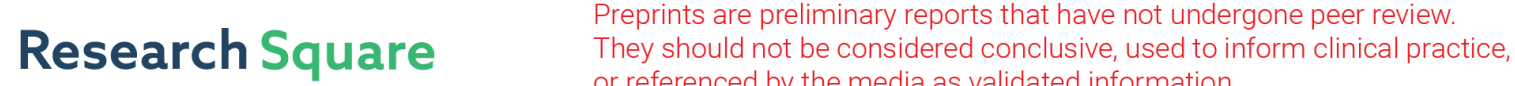 or referenced by the media as validated information. \\ The Classification of Single Traveling Wave Solutions for the Fractional Coupled Nonlinear Schrödinger equation
}

Lu Tang ( $\nabla$ tanglumath@163.com )

Sichuan Normal University https://orcid.org/0000-0002-5051-8364

Shanpeng Chen

Sichuan Normal University

\section{Research Article}

Keywords: Fractional coupled nonlinear Schrödinger equation, Complete discriminant system method, Computer algebra, Traveling wave solution

Posted Date: June 4th, 2021

DOl: https://doi.org/10.21203/rs.3.rs-553359/v1

License: (c) (i) This work is licensed under a Creative Commons Attribution 4.0 International License.

Read Full License

Version of Record: A version of this preprint was published at Optical and Quantum Electronics on January 8th, 2022. See the published version at https://doi.org/10.1007/s11082-021-03496-5. 


\title{
The classification of single traveling wave solutions for the fractional coupled nonlinear Schrödinger equation
}

\author{
Lu Tanga,*, Shanpeng Chen ${ }^{\mathrm{a}}$ \\ ${ }^{a}$ School of Mathematical Science, Sichuan Normal University, Chengdu, 610066, PR China
}

\begin{abstract}
The main purpose of this paper is to study the single traveling wave solutions of the fractional coupled nonlinear Schrödinger equation. By using the complete discriminant system method and computer algebra with symbolic computation, a series of new single traveling wave solutions are obtained, which include trigonometric function solutions, Jacobi elliptic function solutions, hyperbolic function solutions, solitary wave solutions and rational function solutions. In order to further explain the propagation of the fractional coupled nonlinear Schrödinger equation in nonlinear optics, two-dimensional and three-dimensional graphs are drawn.
\end{abstract}

Keywords: Fractional coupled nonlinear Schrödinger equation; Complete discriminant system method; Computer algebra; Traveling wave solution

\section{Introduction}

It is well known that nonlinear evolution equations (NLEEs) model various physical phenomena and play an important position in the investigation of numerous fields, such as combustion theory, fluid dynamics, ecological system, signal processing, nonlinear optics, engineering, statistical 5 mechanics, and plasma physics. As a result, it is one of the critical problems to seek the exact solutions of these NLEEs in nonlinear science. However, due to the complexity of NLEEs, giving all the exact solutions of a NLEE with a unified technique seems to be impossible. Over the decades, a lot of efficient methods have been established and developed to fabricate exact solutions through the efforts of many mathematicians, such as the extended simplest equation method [1, the Riccati sub

\footnotetext{
* Corresponding author

Email address: tanglumath@163.com. (Lu Tang)
} 
equation method [2], the Jacobi elliptic function method [3, 4, Painlevé analysis [5] 9], $\exp _{a}$-function method [10], Lax pairs [11, Bäcklund transformation [12], etc.

In the current study, the fractional coupled nonlinear Schrödinger equation (FCNLSE) is considered in the following form [13, 14

$$
\left\{\begin{array}{l}
i \mathrm{D}_{t}^{\alpha} \psi_{1}+\mathrm{D}_{x}^{2 \beta} \psi_{1}+\delta\left(\left|\psi_{1}\right|^{2}+\gamma\left|\psi_{2}\right|^{2}\right) \psi_{1}=0 \\
i \mathrm{D}_{t}^{\alpha} \psi_{2}+\mathrm{D}_{x}^{2 \beta} \psi_{2}+\delta\left(\gamma\left|\psi_{1}\right|^{2}+\left|\psi_{2}\right|^{2}\right) \psi_{2}=0
\end{array}\right.
$$

where $i$ is imaginary unit, $i^{2}=-1, \alpha, \beta \in(0,1], \psi_{1}=\psi_{1}(x, t)$ and $\psi_{2}=\psi_{2}(x, t)$ are complex functions, which represent the wave amplitudes in two polarizations, $\mathrm{x}$ represents the normalized propagation and t denotes the retard time. $\delta$ represents self-focusing and $\gamma$ denotes cross-phase 15 modulation are nonzero constants. When $\alpha=\beta=1$, it is known to all that Equation 1.1 is the coupled nonlinear Schrödinger equation[15, 16].

The FCNLSE is a classical nonlinear model which can describe lots of physical nonlinear systems. The equation can be applied to many fields, such as biology, fluid mechanics, nonlinear optics, circulation system of chemical industry, heat pulses in solids and so on. Due to the importance of FCNLSE, the equation has been investigated by many researchers [17 22. As a result, it is an important work to seek the exact solutions of the fractional differential equation. So far, lots of effective methods have been established about the traveling wave solutions of the FCNLSE [2326]. In Ref. [27, Esen and his co-workers considered the space-time fractional $(1+1)$-dimensional coupled nonlinear Schrödingger equation, a series of exact solutions including dark, mix dark-bright and nixed singular optical solitons are obtained via the extended sinh-Gordon equation expansion method. By applying the Kudryashov method, the traveling wave solutions to the time FCNLSE were derived by Eslami[28. The traveling wave solutions of Equation (1.1) were obtained by Han and his collaborators [29] by using the bifurcation theory and planar portraits analysis method. Although there are lots of methods to construct the exact solutions of the FCNLSE, the discriminant system method to study the exact solutions of the FCNLSE, it seems as far as we know, not available in the literature. Especially in recent years, with the development of computer algebra theory, by using the mathematical software Maple or Mathematical, a series of traveling wave solutions can be obtained by solving complex algebraic equations. In 1996, with the help of computer algebra, a complete discrimination system of high-order polynomials has been derived by Yang and his co-workers [30. As 35 a matter of fact, it is a powerful tool to seek traveling wave solutions of NLEEs. Therefore, a range 
of solutions of different forms are obtained [31.

In this paper, the complete discriminant system method is employed to seek exact solutions of the FCNLSE, with the assistance of computer algebra and symbolic computation,according to the root-classifications, a series of new traveling wave solutions are obtained.

The organization of this paper is as follows. In section 2, we review the definition of conformable fractional derivatives. In section 3, the description of the complete discriminant system method is given. In section 4, by applying the complete discriminant system method, the new traveling wave solutions to Equation (1.1) are obtained by adapting the inverse transformation. The last section summarizes the results of the current study.

\section{Conformable fractional derivative}

First of all, the conformable fractional derivatives can be defined as follows 32 35.

Definition 2.1. Let $u:[0,+\infty) \rightarrow \mathbf{R}, \alpha \in(0,1]$. The conformable fractional derivative of $u$ of order $\alpha$ is defined as

$$
T_{\alpha}(u)(t)=\lim _{\varepsilon \rightarrow 0} \frac{u\left(t+\varepsilon t^{1-\alpha}\right)-u(t)}{\varepsilon}, \quad \forall t \geq 0,
$$

the function $u$ is $\alpha$-conformable differentiable at a point $t$ if the limit in Equation 2.1) exists.

Theorem 2.2. Assume that $u, v:(0, \infty) \rightarrow R$ be differentiable and also $\alpha$ differentiable functions, then chain rule holds

$$
T_{\alpha}(u \circ v)(t)=\left.t^{1-\alpha} v(t)^{\alpha-1} v^{\prime}(t) T_{\alpha}(u(t))\right|_{t=v(t)} .
$$

\section{Analysis of the method}

Considering the nonlinear partial differential equation in the form:

$$
G\left(u, D_{t}^{\alpha} u, D_{x}^{\beta} u, D_{t}^{\alpha} D_{t}^{\alpha} u, D_{t}^{\alpha} D_{x}^{\beta} u, D_{x}^{\beta} D_{x}^{\beta} u, \ldots\right)=0, \quad 0<\alpha, \beta<1 .
$$

First of all, by traveling wave transformations and some other suitable transformations, the Equation (3.1) can be transferred into an nonlinear ordinary differential equation as follows:

$$
u_{\xi}^{2}=F(u)
$$


50 $\mathrm{s} a_{2}, a_{1}, a_{0}$. Then integrating the above 3.2 , we can obtain

$$
\xi-\xi_{0}=\int \frac{d u}{\sqrt{F(u)}}
$$

where $\xi_{0}$ is the integration constant. Therefore, we need to solve Equation (3.3). However, it is a challenging work to decide the range of the parameters, which can be accomplished by complete discrimination system functions. we can derive its complete discrimination system

$$
\Delta=a_{1}^{2}-4 a_{2} a_{0}
$$

Finally, according to the root-classifications, the parameters mentioned above can be obtained from the integral Equation (3.3). And then by an inverse transformation, we can obtain the exact solutions of the original partial differential equation.

\section{Traveling Wave solutions for FCNLSE}

In this section, we consider the traveling wave solutions for the Equation (1.1), we assume that Equation (1.1) has the following traveling wave transformation

$$
\psi_{1}(x, t)=Z_{1}(\xi) e^{i \eta}, \psi_{2}(x, t)=Z_{2}(\xi) e^{i \eta}, \xi=m\left(\frac{x^{\beta}}{\beta}-c \frac{t^{\alpha}}{\alpha}\right), \eta=-\lambda \frac{x^{\beta}}{\beta}+\mu \frac{t^{\alpha}}{\alpha}+\eta_{0}
$$

where $m, c, \lambda$ and $\mu$ are undetermined real constants, $\eta_{0}$ is an arbitrary constant.

Substituting 4.1 into Equation 1.1, decomposes real parts and imaginary parts of Equation (1.1), the FCNLSE can be reduced into

$$
\left\{\begin{array}{l}
m^{2} Z_{1}^{\prime \prime}+\delta Z_{1}^{3}+\delta \gamma Z_{2}^{2} Z_{1}-\left(\lambda^{2}+\mu\right) Z_{1}=0 \\
m^{2} Z_{2}^{\prime \prime}+\delta Z_{2}^{3}+\delta \gamma Z_{1}^{2} Z_{2}-\left(\lambda^{2}+\mu\right) Z_{2}=0 \\
c=-2 \lambda
\end{array}\right.
$$

Suppose that there is a linear relationship between $Z_{1}$ and $Z_{2}$, namely $Z_{2}=k Z_{1}(k \neq 0)$, substi60 tuting $Z_{2}=k Z_{1}(k \neq 0)$ into the first equation of 4.2 , we can obtain the following form:

$$
m^{2} Z_{1}^{\prime \prime}+\left(\delta+\delta \gamma k^{2}\right) Z_{1}^{3}-\left(\lambda^{2}+\mu\right) Z_{1}=0
$$


Thus,

$$
Z_{1}^{\prime \prime}=-\frac{\delta+\delta \gamma k^{2}}{m^{2}} Z_{1}^{3}+\frac{\lambda^{2}+\mu}{m^{2}} Z_{1}
$$

By multiplying (4.4) with $Z_{1}^{\prime}$, we derive

$$
Z_{1}^{\prime} Z_{1}^{\prime \prime}=-\frac{\delta+\delta \gamma k^{2}}{m^{2}} Z_{1}^{3} Z_{1}^{\prime}+\frac{\lambda^{2}+\mu}{m^{2}} Z_{1} Z_{1}^{\prime} .
$$

Integrating Equation (4.4) once, we obtain

$$
\left(Z_{1}^{\prime}\right)^{2}=a_{4} Z_{1}^{4}+a_{2} Z_{1}^{2}+a_{0}
$$

where $a_{4}=-\frac{\delta+\delta \gamma k^{2}}{2 m^{2}}, a_{2}=\frac{\lambda^{2}+\mu}{m^{2}}, a_{0}$ is a integral constant.

By a suitable transformation as follows

$$
\left\{\begin{array}{l}
Z_{1}= \pm \sqrt{\left(4 a_{4}\right)^{-\frac{1}{3}} W}, \\
b_{1}=4 a_{2}\left(4 a_{4}\right)^{-\frac{2}{3}}, \\
b_{0}=4 a_{0}\left(4 a_{4}\right)^{-\frac{1}{3}}, \\
\xi_{1}=\left(4 a_{4}\right)^{\frac{1}{3}} \xi .
\end{array}\right.
$$

Then the Equation 4.6 can be rewritten as

$$
\left(W_{\xi_{1}}\right)^{2}=W\left(W^{2}+b_{1} W+b_{0}\right)
$$

Integrating Equation (4.8) once, we derive

$$
\pm\left(\xi_{1}-\xi_{0}\right)=\int \frac{d W}{\sqrt{W\left(W^{2}+b_{1} W+b_{0}\right)}},
$$

where $\xi_{0}$ is the integration constant. Denoting $F(W)=W^{2}+b_{1} W+b_{0}$, thus we can establish the second order complete discrimination system as

$$
\Delta=b_{1}^{2}-4 b_{0}
$$

According to the root-classifications of (4.10), there are four cases to be discussed.

Case 1 Suppose that $\Delta=0$. As for $W>0$, we have

$$
\pm\left(\xi_{1}-\xi_{0}\right)=\int \frac{d W}{\left(W+\frac{b_{1}}{2}\right) \sqrt{W}} .
$$


If $b_{1}>0$, it follows from Equation 4.11, we can obtain

$$
W=\frac{b_{1}}{2} \tan ^{2}\left[\frac{1}{2} \sqrt{\frac{b_{1}}{2}}\left(\xi_{1}-\xi_{0}\right)\right] .
$$

According to the Equation (4.7), 4.12) and $Z_{2}=k Z_{1}(k \neq 0)$, the solution of Equation 1.1) can be obtained as follows (see Figure 1)

$$
\left\{\begin{aligned}
\psi_{1,1}(x, t)= & \pm \sqrt{\frac{\lambda^{2}+\mu}{-\delta-\delta \gamma k^{2}}} \tan \left\{2^{-\frac{7}{6}} \sqrt{\frac{\lambda^{2}+\mu}{m^{2}}}\left(\frac{2 m^{2}}{-\delta-\delta \gamma k^{2}}\right)^{1 / 3}\left[\left(\frac{-2 \delta-2 \delta \gamma k^{2}}{m^{2}}\right)^{1 / 3} \xi-\xi_{0}\right]\right\} \\
& \times \exp \left(i\left(-\lambda \frac{x^{\beta}}{\beta}+\mu \frac{t^{\alpha}}{\alpha}+\eta_{0}\right)\right), \\
\psi_{2,1}(x, t)= & \pm k \sqrt{\frac{\lambda^{2}+\mu}{-\delta-\delta \gamma k^{2}}} \tan \left\{2^{-\frac{7}{6}} \sqrt{\frac{\lambda^{2}+\mu}{m^{2}}}\left(\frac{2 m^{2}}{-\delta-\delta \gamma k^{2}}\right)^{1 / 3}\left[\left(\frac{-2 \delta-2 \delta \gamma k^{2}}{m^{2}}\right)^{1 / 3} \xi-\xi_{0}\right]\right\} \\
& \times \exp \left(i\left(-\lambda \frac{x^{\beta}}{\beta}+\mu \frac{t^{\alpha}}{\alpha}+\eta_{0}\right)\right) .
\end{aligned}\right.
$$

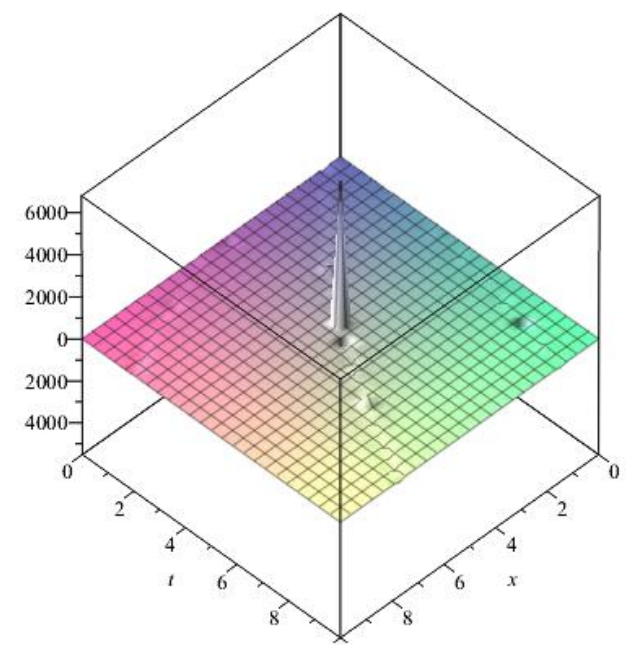

(a) Real 3D surface

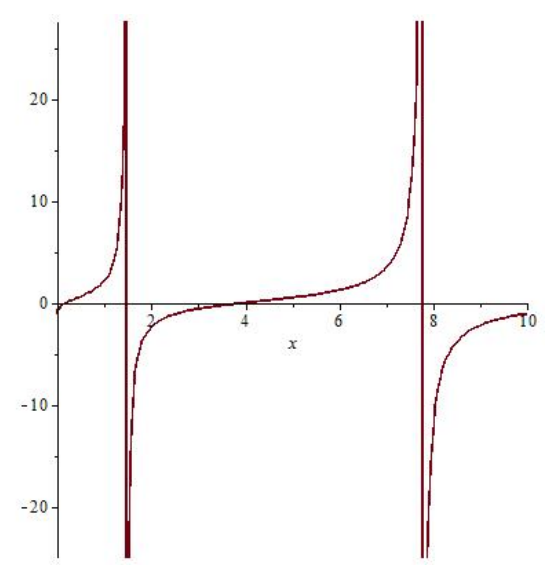

(b) Real 2D Shape

Figure 1: The graphics of $\psi_{1,1}(x, t)$ in Equation 4.13 at $\lambda=\mu=\gamma=k=m=1, c=2, \delta=-1, \xi_{0}=0, \alpha=\beta=\frac{1}{2}$.

If $b_{1}<0$, it follows from Equation (4.11), we can obtain

$$
\left\{\begin{array}{l}
W=-\frac{b_{1}}{2} \tanh ^{2}\left[\sqrt{-\frac{b_{1}}{2}} \times \frac{1}{2}\left(\xi_{1}-\xi_{0}\right)\right], \\
W=-\frac{b_{1}}{2} \operatorname{coth}^{2}\left[\sqrt{-\frac{b_{1}}{2}} \times \frac{1}{2}\left(\xi_{1}-\xi_{0}\right)\right] .
\end{array}\right.
$$


According to the Equation (4.7), (4.14) and $Z_{2}=k Z_{1}(k \neq 0)$, the solution of Equation (1.1) can be obtained as follows (see Figure 2 and Figure 3 )

$$
\left\{\begin{aligned}
\psi_{1,2}(x, t)= & \pm \sqrt{\frac{\lambda^{2}+\mu}{\delta+\delta \gamma k^{2}}} \tanh \left\{\sqrt{\frac{\lambda^{2}+\mu}{-2 m^{2}}}\left(\frac{m^{2}}{-2 \delta-2 \delta \gamma k^{2}}\right)^{1 / 3}\left[\left(\frac{-2 \delta-2 \delta \gamma k^{2}}{m^{2}}\right)^{1 / 3} \xi-\xi_{0}\right]\right\} \\
& \times \exp \left(i\left(-\lambda \frac{x^{\beta}}{\beta}+\mu \frac{t^{\alpha}}{\alpha}+\eta_{0}\right)\right), \\
\psi_{2,2}(x, t)= & \pm k \sqrt{\frac{\lambda^{2}+\mu}{\delta+\delta \gamma k^{2}}} \tanh \left\{\sqrt{\frac{\lambda^{2}+\mu}{-2 m^{2}}}\left(\frac{m^{2}}{-2 \delta-2 \delta \gamma k^{2}}\right)^{1 / 3}\left[\left(\frac{-2 \delta-2 \delta \gamma k^{2}}{m^{2}}\right)^{1 / 3} \xi-\xi_{0}\right]\right\} \\
& \times \exp \left(i\left(-\lambda \frac{x^{\beta}}{\beta}+\mu \frac{t^{\alpha}}{\alpha}+\eta_{0}\right)\right) .
\end{aligned}\right.
$$

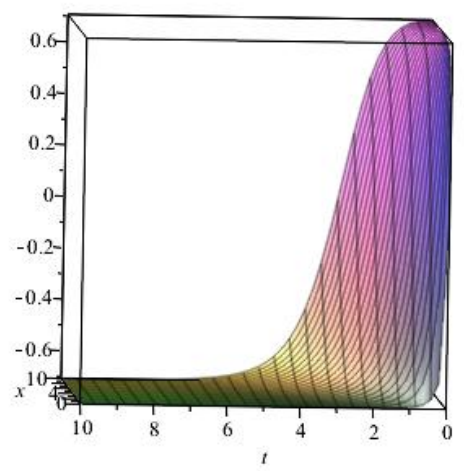

(a) Real 3D surface

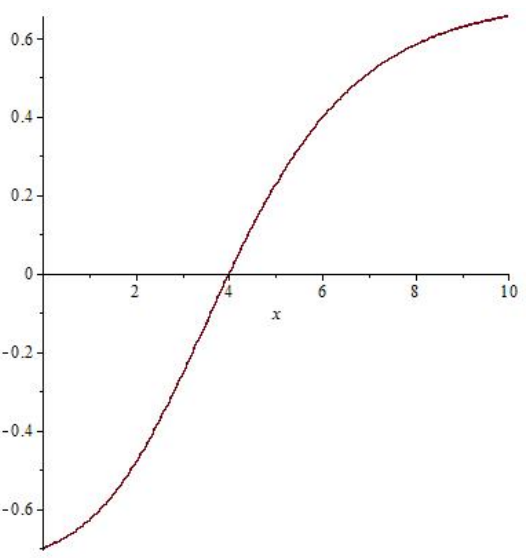

(b) Real 2D Shape

Figure 2: The graphics of $\psi_{1,2}(x, t)$ in Equation 4.15 at $\lambda=\gamma=k=m=1, \mu=-2, \delta=-1, c=2, \xi_{0}=0$, $\alpha=\beta=\frac{1}{2}$.

$$
\left\{\begin{aligned}
\psi_{1,3}(x, t)= & \pm \sqrt{\frac{\lambda^{2}+\mu}{\delta+\delta \gamma k^{2}}} \operatorname{coth}\left\{\sqrt{\frac{\lambda^{2}+\mu}{-2 m^{2}}}\left(\frac{m^{2}}{-2 \delta-2 \delta \gamma k^{2}}\right)^{1 / 3}\left[\left(\frac{-2 \delta-2 \delta \gamma k^{2}}{m^{2}}\right)^{1 / 3} \xi-\xi_{0}\right]\right\} \\
& \times \exp \left(i\left(-\lambda \frac{x^{\beta}}{\beta}+\mu \frac{t^{\alpha}}{\alpha}+\eta_{0}\right)\right) \\
\psi_{2,3}(x, t)= & \pm k \sqrt{\frac{\lambda^{2}+\mu}{\delta+\delta \gamma k^{2}}} \operatorname{coth}\left\{\sqrt{\frac{\lambda^{2}+\mu}{-2 m^{2}}}\left(\frac{m^{2}}{-2 \delta-2 \delta \gamma k^{2}}\right)^{1 / 3}\left[\left(\frac{-2 \delta-2 \delta \gamma k^{2}}{m^{2}}\right)^{1 / 3} \xi-\xi_{0}\right]\right\} \\
& \times \exp \left(i\left(-\lambda \frac{x^{\beta}}{\beta}+\mu \frac{t^{\alpha}}{\alpha}+\eta_{0}\right)\right) .
\end{aligned}\right.
$$

If $b_{1}=0$, it follows from Equation 4.11, we can obtain

$$
W=\frac{4}{\left(\xi_{1}-\xi_{0}\right)^{2}} .
$$




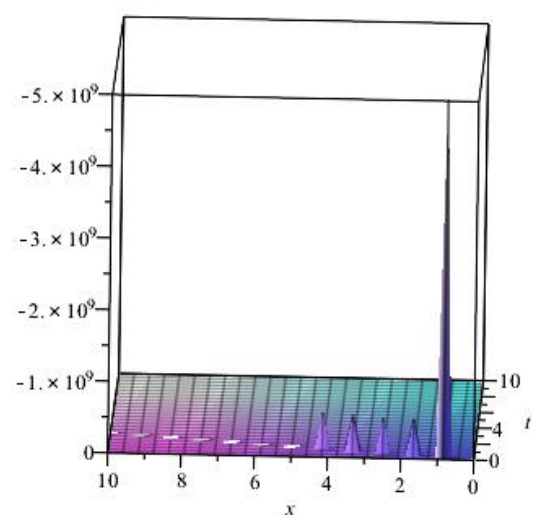

(a) Real 3D surface

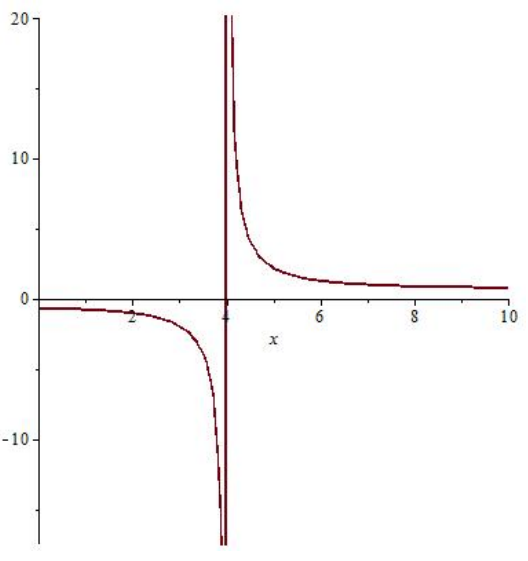

(b) Real 2D Shape

Figure 3: The graphics of $\psi_{1,3}(x, t)$ in Equation 4.16 at $\lambda=\gamma=k=m=1, \mu=-2, \delta=-1, c=2, \xi_{0}=0$, $\alpha=\beta=\frac{1}{2}$.

Thus, the solution of Equation (1.1) can be obtained as follows (see Figure 4)

$$
\left\{\begin{aligned}
\psi_{1,4}(t, x)= & \pm 2^{\frac{2}{3}}\left(\frac{-\delta-\delta \gamma k^{2}}{2 m^{2}}\right)^{-1 / 6}\left[\left(\frac{-2 \delta-2 \delta \gamma k^{2}}{m^{2}}\right) \xi-\xi_{0}\right] \\
& \times \exp \left(i\left(-\lambda \frac{x^{\beta}}{\beta}+\mu \frac{t^{\alpha}}{\alpha}+\eta_{0}\right)\right), \\
\psi_{2,4}(t, x)= & \pm 2^{\frac{2}{3}} k\left(\frac{-\delta-\delta \gamma k^{2}}{2 m^{2}}\right)^{-1 / 6}\left[\left(\frac{-2 \delta-2 \delta \gamma k^{2}}{m^{2}}\right) \xi-\xi_{0}\right] \\
& \times \exp \left(i\left(-\lambda \frac{x^{\beta}}{\beta}+\mu \frac{t^{\alpha}}{\alpha}+\eta_{0}\right)\right) .
\end{aligned}\right.
$$

Case 2 Suppose that $\Delta>0$ and $b_{0}=0$. As for $W>-b_{1}$, we have

$$
\pm\left(\xi_{1}-\xi_{0}\right)=\int \frac{d W}{W \sqrt{W+b_{1}}} .
$$

If $b_{1}>0$, it follows from Equation 4.19, we can obtain

$$
\left\{\begin{array}{l}
W=\frac{b_{1}}{2} \tanh ^{2}\left[\sqrt{\frac{b_{1}}{2}} \times \frac{1}{2}\left(\xi_{1}-\xi_{0}\right)\right]-b_{1}, \\
W=\frac{b_{1}}{2} \operatorname{coth}^{2}\left[\sqrt{\frac{b_{1}}{2}} \times \frac{1}{2}\left(\xi_{1}-\xi_{0}\right)\right]-b_{1} .
\end{array}\right.
$$

According to the Equation 4.7), 4.20 and $Z_{2}=k Z_{1}(k \neq 0)$, the solution of Equation 1.1 can 


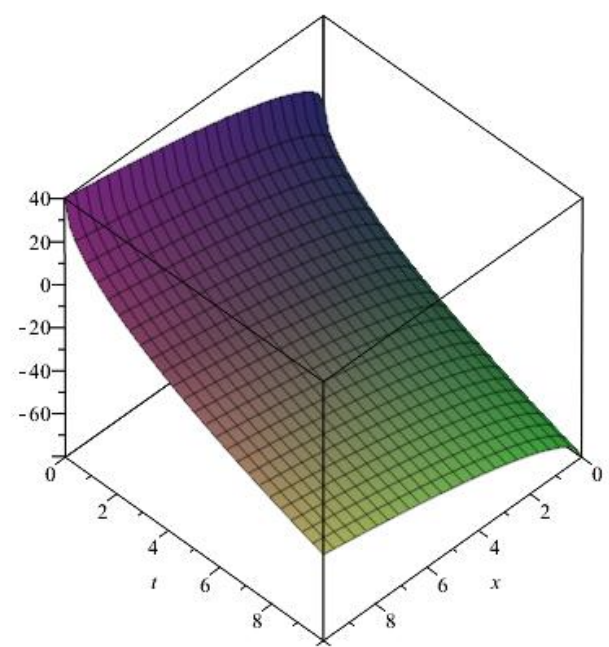

(a) Real 3D surface

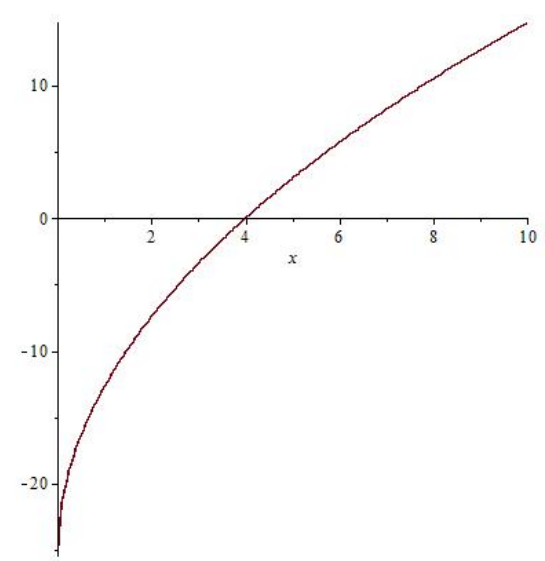

(b) Real 2D Shape

Figure 4: The graphics of $\psi_{1,4}(x, t)$ in Equation 4.18 at $\gamma=k=m=1, \delta=-1, c=2, \xi_{0}=0, \alpha=\beta=\frac{1}{2}$.

be obtained as follows:

$$
\begin{aligned}
& \left\{\begin{aligned}
\psi_{1,5}(x, t)= & \pm\left(\frac{-2 \delta-2 \delta \gamma k^{2}}{m^{2}}\right)^{-1 / 6}\left\{\left(\frac{2 \lambda^{2}+2 \mu}{m^{2}}\right)\left(\frac{-2 \delta-2 \delta \gamma k^{2}}{m^{2}}\right)^{-\frac{2}{3}}\right. \\
& \left.\left.\times \tanh ^{2}\left[\frac{\sqrt{2}}{2} \sqrt{\frac{\lambda^{2}+\mu}{m^{2}}}\left(\frac{-2 \delta-2 \delta \gamma k^{2}}{m^{2}}\right)^{-1 / 3} \xi-\xi_{0}\right)\right]-\left(\frac{4 \lambda^{2}+4 \mu}{m^{2}}\right)\left(\frac{-2 \delta-2 \delta \gamma k^{2}}{m^{2}}\right)^{-\frac{2}{3}}\right\}^{\frac{1}{2}} \\
& \times \exp \left(i\left(-\lambda \frac{x^{\beta}}{\beta}+\mu \frac{t^{\alpha}}{\alpha}+\eta_{0}\right)\right), \\
\psi_{2,5}(x, t)= & \pm k\left(\frac{-2 \delta-2 \delta \gamma k^{2}}{m^{2}}\right)^{-1 / 6}\left\{\left(\frac{2 \lambda^{2}+2 \mu}{m^{2}}\right)\left(\frac{-2 \delta-2 \delta \gamma k^{2}}{m^{2}}\right)^{-\frac{2}{3}}\right. \\
& \left.\left.\times \tanh ^{2}\left[\frac{\sqrt{2}}{2} \sqrt{\frac{\lambda^{2}+\mu}{m^{2}}}\left(\frac{-2 \delta-2 \delta \gamma k^{2}}{m^{2}}\right)^{-1 / 3} \xi-\xi_{0}\right)\right]-\left(\frac{4 \lambda^{2}+4 \mu}{m^{2}}\right)\left(\frac{-2 \delta-2 \delta \gamma k^{2}}{m^{2}}\right)^{-\frac{2}{3}}\right\}^{\frac{1}{2}} \\
& \times \exp \left(i\left(-\lambda \frac{x^{\beta}}{\beta}+\mu \frac{t^{\alpha}}{\alpha}+\eta_{0}\right)\right) .
\end{aligned}\right. \\
& \left\{\begin{aligned}
\psi_{1,6}(x, t)= & \pm\left(\frac{-2 \delta-2 \delta \gamma k^{2}}{m^{2}}\right)^{-1 / 6}\left\{\left(\frac{2 \lambda^{2}+2 \mu}{m^{2}}\right)\left(\frac{-2 \delta-2 \delta \gamma k^{2}}{m^{2}}\right)^{-\frac{2}{3}}\right. \\
& \left.\left.\times \operatorname{coth}^{2}\left[\frac{\sqrt{2}}{2} \sqrt{\frac{\lambda^{2}+\mu}{m^{2}}}\left(\frac{-2 \delta-2 \delta \gamma k^{2}}{m^{2}}\right)^{-1 / 3} \xi-\xi_{0}\right)\right]-\left(\frac{4 \lambda^{2}+4 \mu}{m^{2}}\right)\left(\frac{-2 \delta-2 \delta \gamma k^{2}}{m^{2}}\right)^{-\frac{2}{3}}\right\}^{\frac{1}{2}} \\
& \times \exp \left(i\left(-\lambda \frac{x^{\beta}}{\beta}+\mu \frac{t^{\alpha}}{\alpha}+\eta_{0}\right)\right), \\
\psi_{2,6}(x, t)= & \pm k\left(\frac{-2 \delta-2 \delta \gamma k^{2}}{m^{2}}\right)^{-1 / 6}\left\{\left(\frac{2 \lambda^{2}+2 \mu}{m^{2}}\right)\left(\frac{-2 \delta-2 \delta \gamma k^{2}}{m^{2}}\right)^{-\frac{2}{3}}\right. \\
& \left.\left.\times \operatorname{coth}^{2}\left[\frac{\sqrt{2}}{2} \sqrt{\frac{\lambda^{2}+\mu}{m^{2}}}\left(\frac{-2 \delta-2 \delta \gamma k^{2}}{m^{2}}\right)^{-1 / 3} \xi-\xi_{0}\right)\right]-\left(\frac{4 \lambda^{2}+4 \mu}{m^{2}}\right)\left(\frac{-2 \delta-2 \delta \gamma k^{2}}{m^{2}}\right)^{-\frac{2}{3}}\right\}^{\frac{1}{2}} \\
& \times \exp \left(i\left(-\lambda \frac{x^{\beta}}{\beta}+\mu \frac{t^{\alpha}}{\alpha}+\eta_{0}\right)\right) .
\end{aligned}\right.
\end{aligned}
$$


If $b_{1}<0$, it follows from Equation 4.19 , we can obtain

$$
W=-\frac{b_{1}}{2} \tan ^{2}\left[\sqrt{-\frac{b_{1}}{2}} \times \frac{1}{2}\left(\xi_{1}-\xi_{0}\right)\right]-b_{1} .
$$

According to the Equation (4.7), (4.23) and $Z_{2}=k Z_{1}(k \neq 0)$, the solution of Equation (1.1) can be obtained as follows:

$$
\left\{\begin{aligned}
\psi_{1,7}(x, t)= & \pm\left(\frac{-2 \delta-2 \delta \gamma k^{2}}{m^{2}}\right)^{-1 / 6}\left\{\left(\frac{-2 \lambda^{2}-2 \mu}{m^{2}}\right)\left(\frac{-2 \delta-2 \delta \gamma k^{2}}{m^{2}}\right)^{-\frac{2}{3}}\right. \\
& \left.\left.\times \tan ^{2}\left[\frac{\sqrt{2}}{2} \sqrt{-\frac{\lambda^{2}+\mu}{m^{2}}}\left(\frac{-2 \delta-2 \delta \gamma k^{2}}{m^{2}}\right)^{-1 / 3} \xi-\xi_{0}\right)\right]-\left(\frac{4 \lambda^{2}+4 \mu}{m^{2}}\right)\left(\frac{-2 \delta-2 \delta \gamma k^{2}}{m^{2}}\right)^{-\frac{2}{3}}\right\}^{\frac{1}{2}} \\
& \times \exp \left(i\left(-\lambda \frac{x^{\beta}}{\beta}+\mu \frac{t^{\alpha}}{\alpha}+\eta_{0}\right)\right), \\
\psi_{2,7}(x, t)= & \pm k\left(\frac{-2 \delta-2 \delta \gamma k^{2}}{m^{2}}\right)^{-1 / 6}\left\{\left(\frac{-2 \lambda^{2}-2 \mu}{m^{2}}\right)\left(\frac{-2 \delta-2 \delta \gamma k^{2}}{m^{2}}\right)^{-\frac{2}{3}}\right. \\
& \left.\left.\times \tan ^{2}\left[\frac{\sqrt{2}}{2} \sqrt{-\frac{\lambda^{2}+\mu}{m^{2}}}\left(\frac{-2 \delta-2 \delta \gamma k^{2}}{m^{2}}\right)^{-1 / 3} \xi-\xi_{0}\right)\right]-\left(\frac{4 \lambda^{2}+4 \mu}{m^{2}}\right)\left(\frac{-2 \delta-2 \delta \gamma k^{2}}{m^{2}}\right)^{-\frac{2}{3}}\right\}^{\frac{1}{2}} \\
& \times \exp \left(i\left(-\lambda \frac{x^{\beta}}{\beta}+\mu \frac{t^{\alpha}}{\alpha}+\eta_{0}\right)\right) .
\end{aligned}\right.
$$

Case 3 Suppose that $\Delta>0$ and $b_{0} \neq 0$ and $\omega_{1}<\omega_{2}<\omega_{3}$. Then, we adopt the assumption that one of $\omega_{1}, \omega_{2}, \omega_{3}$ is zero and the rest of them are two different real roots of $F(W)=0$. Taking the transformation $W=\omega_{1}+\left(\omega_{2}-\omega_{1}\right) \sin ^{2} \theta$, it is clear that

$$
\pm\left(\xi_{1}-\xi_{0}\right)=\frac{1}{\sqrt{\omega_{3}-\omega_{1}}} \int \frac{d \theta}{\sqrt{1-n_{1}^{2} \sin ^{2} \theta}},
$$

where $n_{1}^{2}=\frac{\omega_{2}-\omega_{1}}{\omega_{3}-\omega_{1}}$. It follows from Equation 4.25, we obtain

$$
W=\omega_{1}+\left(\omega_{2}-\omega_{1}\right) \operatorname{sn}^{2}\left(\sqrt{\omega_{3}-\omega_{1}}\left(\frac{1}{2}\left(\xi_{1}-\xi_{0}\right)\right), n_{1}\right) .
$$

According to the Equation (4.7), 4.26) and $Z_{2}=k Z_{1}(k \neq 0)$, the solution of Equation (1.1) can be obtained as follows

$$
\left\{\begin{aligned}
\psi_{1,8}(x, t)= & \pm\left(\frac{-2 \delta-2 \delta \gamma k^{2}}{m^{2}}\right)^{-\frac{1}{6}}\left\{\omega_{1}+\left(\omega_{3}-\omega_{1}\right) s n^{2}\left[\frac{\sqrt{\omega_{3}-\omega_{1}}}{2}\left(\left(\frac{-2 \delta-2 \delta \gamma k^{2}}{m^{2}}\right) \xi-\xi_{0}\right), n_{1}\right]\right\}^{\frac{1}{2}} \\
& \times \exp \left(i\left(-\lambda \frac{x^{\beta}}{\beta}+\mu \frac{t^{\alpha}}{\alpha}+\eta_{0}\right)\right), \\
\psi_{2,8}(x, t)= & \pm k\left(\frac{-2 \delta-2 \delta \gamma k^{2}}{m^{2}}\right)^{-\frac{1}{6}}\left\{\omega_{1}+\left(\omega_{3}-\omega_{1}\right) s n^{2}\left[\frac{\sqrt{\omega_{3}-\omega_{1}}}{2}\left(\left(\frac{-2 \delta-2 \delta \gamma k^{2}}{m^{2}}\right) \xi-\xi_{0}\right), n_{1}\right]\right\}^{\frac{1}{2}} \\
& \times \exp \left(i\left(-\lambda \frac{x^{\beta}}{\beta}+\mu \frac{t^{\alpha}}{\alpha}+\eta_{0}\right)\right) .
\end{aligned}\right.
$$

For another transformation $W=\frac{-\omega_{2} \sin ^{2} \theta+\omega_{3}}{\cos ^{2} \theta}$, it follows from Equation 4.25, we obtain

$$
W=\frac{-\omega_{2} \operatorname{sn}\left(\sqrt{\omega_{3}-\omega_{1}}\left(\frac{1}{2}\left(\xi_{1}-\xi_{0}\right)\right), n_{1}\right)+\omega_{3}}{c n\left(\sqrt{\omega_{3}-\omega_{1}}\left(\frac{1}{2}\left(\xi_{1}-\xi_{0}\right)\right), n_{1}\right)}
$$


According to the Equation (4.7), 4.28) and $Z_{2}=k Z_{1}(k \neq 0)$, the solution of Equation (1.1) can be obtained as follows:

$$
\left\{\begin{aligned}
\psi_{1,9}(x, t)= & \pm\left(\frac{-2 \delta-2 \delta \gamma k^{2}}{m^{2}}\right)^{-\frac{1}{6}}\left\{\frac{-\omega_{2} s n\left[\frac{\sqrt{\omega_{3}-\omega_{1}}}{2}\left(\left(\frac{-2 \delta-2 \delta \gamma k^{2}}{m^{2}}\right)^{1 / 3} \xi-\xi_{0}\right), n_{1}\right]+\omega_{3}}{c n\left[\frac{\sqrt{\omega_{3}-\omega_{1}}}{2}\left(\left(\frac{-2 \delta-2 \delta \gamma k^{2}}{m^{2}}\right)^{1 / 3} \xi-\xi_{0}\right), n_{1}\right]}\right\}^{\frac{1}{2}} \\
& \times \exp \left(i\left(-\lambda \frac{x^{\beta}}{\beta}+\mu \frac{t^{\alpha}}{\alpha}+\eta_{0}\right)\right) . \\
\psi_{2,9}(x, t)= & \pm k\left(\frac{-2 \delta-2 \delta \gamma k^{2}}{m^{2}}\right)^{-\frac{1}{6}}\left\{\frac{-\omega_{2} s n\left[\frac{\sqrt{\omega_{3}-\omega_{1}}}{2}\left(\left(\frac{-2 \delta-2 \delta \gamma k^{2}}{m^{2}}\right)^{1 / 3} \xi-\xi_{0}\right), n_{1}\right]+\omega_{3}}{c n\left[\frac{\sqrt{\omega_{3}-\omega_{1}}}{2}\left(\left(\frac{-2 \delta-2 \delta \gamma k^{2}}{m^{2}}\right)^{1 / 3} \xi-\xi_{0}\right), n_{1}\right]}\right\}^{\frac{1}{2}} \\
& \times \exp \left(i\left(-\lambda \frac{x^{\beta}}{\beta}+\mu \frac{t^{\alpha}}{\alpha}+\eta_{0}\right)\right) .
\end{aligned}\right.
$$

Case 4 Suppose that $\Delta<0$. Taking the transformation $W=\sqrt{b_{0}} \tan ^{2} \frac{\theta}{2}$, it is clear that

$$
\pm\left(\xi_{1}-\xi_{0}\right)=\frac{1}{2}\left(b_{0}\right)^{-\frac{1}{4}} \int \frac{d \theta}{\sqrt{1-n_{2}^{2} \sin ^{2} \theta}} .
$$

where $n_{2}^{2}=\frac{2 \sqrt{b_{0}-b_{1}}}{4 \sqrt{b_{0}}}$, it follows from Equation 4.30 , we obtain

$$
W=\frac{2 \sqrt{b_{0}}}{1+\operatorname{cn}\left(b_{0}^{\frac{1}{4}}\left(\xi_{1}-\xi_{0}\right), n_{2}\right)}-\sqrt{b_{0}} .
$$

According to the Equation (4.7), 4.31) and $Z_{2}=k Z_{1}(k \neq 0)$, the solution of Equation 1.1) can be obtained as follows

$$
\left\{\begin{aligned}
\psi_{1,10}(x, t)= & \pm\left(\frac{-2 \delta-2 \delta \gamma k^{2}}{m^{2}}\right)^{-\frac{1}{6}}\left\{\frac{2 \sqrt{b_{0}}}{1+c n\left[b_{0}^{\frac{1}{4}}\left(\left(\frac{-2 \delta-2 \delta \gamma k^{2}}{m^{2}}\right)^{\frac{1}{3}} \xi-\xi_{0}\right), n_{2}\right]}-\sqrt{b_{0}}\right\}^{\frac{1}{2}} \\
& \times \exp \left(i\left(-\lambda \frac{x^{\beta}}{\beta}+\mu \frac{t^{\alpha}}{\alpha}+\eta_{0}\right)\right), \\
\psi_{2,10}(x, t)= & \pm k\left(\frac{-2 \delta-2 \delta \gamma k^{2}}{m^{2}}\right)^{-\frac{1}{6}}\left\{\frac{2 \sqrt{b_{0}}}{1+c n\left[b_{0}^{\frac{1}{4}}\left(\left(\frac{-2 \delta-2 \delta k^{2}}{m^{2}}\right)^{\frac{1}{3}} \xi-\xi_{0}\right), n_{2}\right]}-\sqrt{b_{0}}\right\}^{\frac{1}{2}} \\
& \times \exp \left(i\left(-\lambda \frac{x^{\beta}}{\beta}+\mu \frac{t^{\alpha}}{\alpha}+\eta_{0}\right)\right) .
\end{aligned}\right.
$$

\section{Conclusion}

It is known to all that the nonlinear Schrödinger equation is an example of a universal nonlinear model that describes many physical nonlinear systems. In this paper, the complete discriminant system method is employed to seek exact solutions of the FCNLSE, by using the mathematical 
75

ware Maple, combining computer algebra with symbolic computation, we obtain a series of new traveling wave solutions, including trigonometric function solutions, Jacobi elliptic function solutions, hyperbolic function solutions, solitary wave solutions, rational function solutions. The complete discriminant system method is employed to seek traveling wave solutions of CFNLSE, it seems as far as we know, not available in the literature. Therefore, the research in this paper has an

\section{Funding}

This work was supported by the Specialized Research Fund for the Doctoral Program of Higher Education of China under grant No.20115134110001.

\section{Authors' contributions}

All authors read and proved the final manuscript.

\section{References}

[1] M.E. Elsayed, M.A. Reham, A. Biswas, et al. Dispersive solitons in optical fibers and DWDM networks with Schrödinger-Hirota equation. Optik. 199 (2019) 163214.

[2] F.S. Khodadad, F. Nazari, et al. Soliton solutions of the conformable fractional ZakharovKuznetsov equation with dual-power law nonlinearity. Opt. Quant. Electron 49 (2017) 384.

[3] E.J. Parks, B.R. Duffy, P.C. Abbott. The Jacobi elliptic function method for fingding periodic wave solutions to nonlinear evolution equations. Phys. Lett. A 295 (2002) 280-286.

[4] F. Tchier, E.C. Aslan, M. Inc. Optical solitons in parabplic law medium: Jacobi elliptic function solution. Nonlinear Dyn. 85 (2016) 2577-2582.

95

[5] T. Bountis, P. Vanhaecke. Lotka-Volterra systems satisfying a strong painlevé property. Phys. Lett. A 380 (2016) 3977-3982.

[6] V. Marinakis, T. Bountis. Special solutions of a new class of water wave equations. Comm. Appl. Anal. 4 (2000) 433-445. 
[7] E. Tzirtzilakis, M. Xenos, V. Marinakis, T. Bountis. Interactions and stability of solitary waves in shallow water. Chaos. Solitons and Fractals. 14 (2002) 87-95.

[8] E. Tzirtzilakis, V. Marinakis, C. Apokis, T. Bountis. Soliton-like solutions of higer order water wave equations of the Kdv type. J. Math. Phys. 43 (2002) 6151-6165.

[9] J. Weiss, M. Tabor, G. Carnevale. The Painlevé property for partial differential equations. J. Math. Phys. 24 (1983) 522-526.

[10] K. Hosseini, R. Ansari, F. Samadani, et al. High-order disperive cuboc-quintic Schrödinger equation and its exact solutions. Optik. 136 (2019) 203-207.

[11] W.X. Ma, W. Strampp. An explicit symmetry constraint for the Lax pairs and the adjoint Lax pairs of AKNS systems. Phys. Lett. A 185 (1999) 277-286.

[12] Y, Huang. New no-traveling wave solutions for the Liouville equation by Bäcklund transformation method. Nonlinear Dyn. 71 (2013) 87-90.

[13] Y.J. Zhang, C.Y. Yang, W.T. Yu, et al. Intercations of vector anti-dark solitons for the coupled nonlinear Schrödinger equation in inhomogeneous fibers. Nonlinear Dyn. 94 (2018) 1351-1360.

[14] R. M. EI-Shiekh, M. Gaballah. Solitary wave solutions for the variable-coefficient coupled nonlinear Schrödinger equations and Davey-Stewartson system using modified sine-Gordon equation method. J. Ocean Eng. Sci 5 (2020) 180.

[15] T. A. Sulaiman, H. Bulut. Optical solitons and modulation instability analysis of the $(1+1)-$ dimensional coupled nonlinear Schrödinger equation. Commun. Theor. Phys 72 (2020) 025003.

[16] R. W. Boyd, Nonlinear Optics. Academic, San Diego, 1992.

[17] M. Younis. The first integral method for time-space fractional differential equations. J. Adv. Phys. 2 (2013) 220-223.

[18] A. Bekir, O. Guner, A. H. Bhrawy, A, Biswas. Solving nonlinear frational differential equations using exp-function and $\frac{G^{\prime}}{G}$-expansion methods. Rom. J. Phys. 60 (2015) 360-378.

[19] Z.S. Wen. The generalized bifurcation method for deriving exact solutions of nonlinear spacetime fractional partial differential equations Appl. Math. Comput. 366 (2020) 124735. 
[20] L.X. Du, Y.H. Sun, D.S. Wu. Bifurcations and solutions for the generalized nonlinear Schrödinger equation. Phys. Lett. A 383 (2019) 126028.

[21] Z.Y. Zhang, Z.H. Liu, X.J. Miao, et al. Qualitative analysis and traveling wave solutions for the perturbed nonlienar Schrödinger equation with Kerr law nonlienarity. Phys. Lett. A 375 (2011) 1275 .

[22] Q. Guo, J. Liu. New exact solutions to the nonlienar Schrödinger equation with variable coefficients. Results Phys 16 (2020) 102857.

[23] T.A.Gadzhimuradov, A.M.Agalarov, R.Radha, B.Tamil Arasan. Dynamics of solitons in the fourth-order nonlocal nonlinear Schrödinger equation. Nonlinear Dyn. 99 (2020) 1295-1300.

[24] Y.Y. Xie, Z.Y. Yang, L.F. Li. New exact solutions to the high dispersive cubic-quintic nonlinear Schrödinger equation. Phys. Lett. A. 382 (2018) 2506-2514.

[25] Y. Li, D.C. Lu, M. Arshad, X. Xu. New exact traveling wave solutons of the unstable nonlinar Schrödinger equations and their applications. Optik. 226 (2021) 165386.

[26] R. M. EI-Shiekh. Classes of new exact solutions for nonlienar Schrödinger equations with variable coefficients arising in optical fiber. Results Phys. 13 (2019) 102214.

[27] A. Esen, T. A. Sulaiman, et al. Optical solitons to the space-time fractional $(1+1)$-dimensional coupled nonlinear Schrödinger equation. Optik. 167 (2018) 150-156.

[28] M. Eslami. Exact traveling wave solutions to the fractional coupled nonlinear Schrodinger equations. Appl. Math. Comput 285 (2016) 141-148.

[29] T.Y. Han, Z, Li, X, Zhang. Bifurcation and new exact traveling wave solutions to time-space coupled fractional nonlinear Schrödinger equation. Phys. Lett. A. 395 (2021) 127217.

[30] L. Yang, X.Y. Hou, Z.B. Zeng. Compete discrimation system for polynomial. Sci China Ser E. 26 (1996) 628-646.

[31] Y. Zheng, S.Y. Lai. Peakons, solitary patterns and periodic solutions for generalized CamassaHolm equations. Phys. Lett. A. 372 (2008) 4141-4143. 
[32] C. Chen, Y.L. Jiang, Z.L. Wang, et al, Bifurcation behavior and exact solutions for timefractional nonlinear Schrödinger equation with parabolic law nonlinearity. Optik, 222 (2020) 165331.

[33] L. Tang, Exact solutions to conformable time-fractional Klein-Gordon equation with high-order nonlinearities. Results Phys 18 (2020) 103289.

[34] M,A. Hammad, R. Khalil. Conformable fractional heat differential equation. Int. J. Pure Appl. Math. 94 (2014) 215-221.

[35] R. Khalil, A. Horani, A. Yousef, et al. A new definition of fractional derivative. J. Comp. Appl. Math. 264 (2014) 65-70. 
Figures

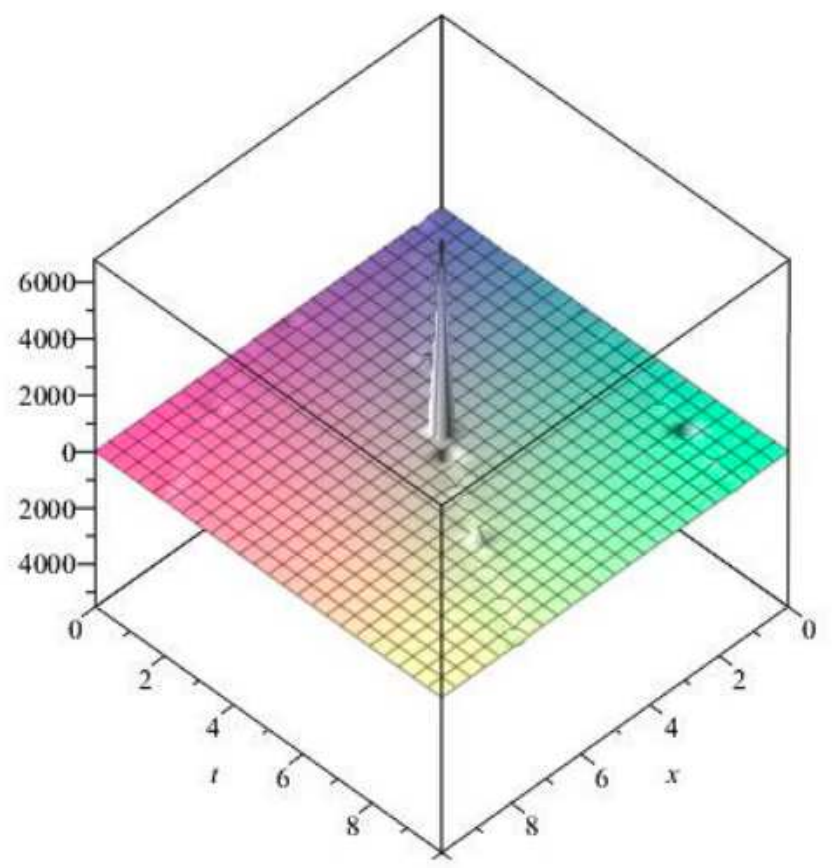

(a) Real 3D surface

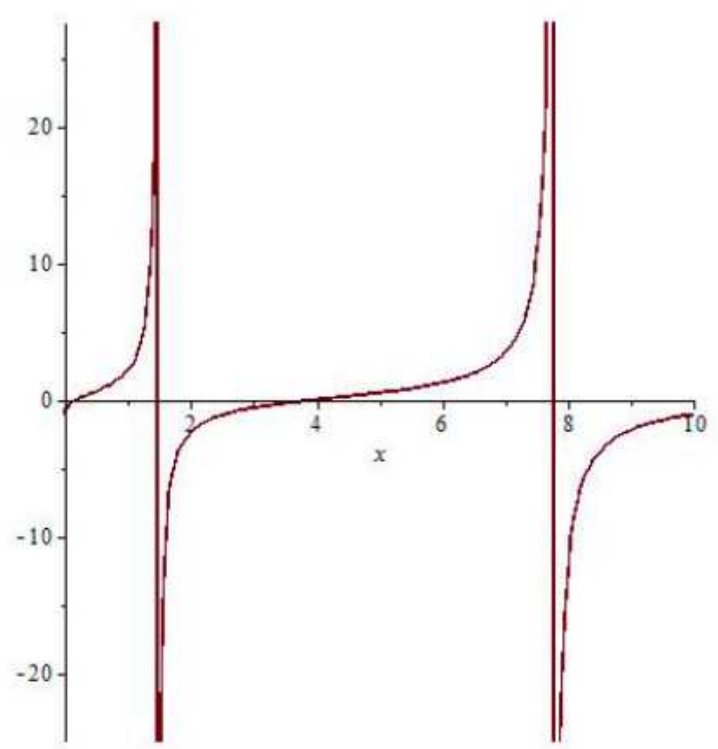

(b) Real 2D Shape

\section{Figure 1}

Please see the Manuscript PDF file for the complete figure caption

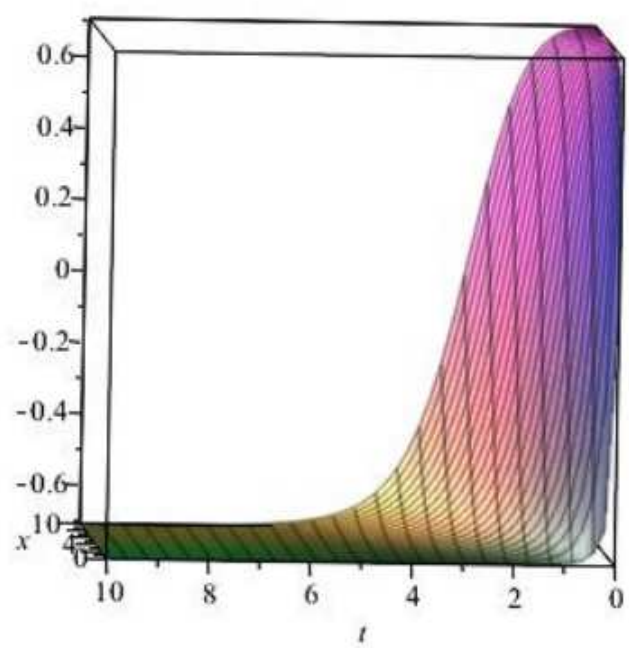

(a) Real 3D surface

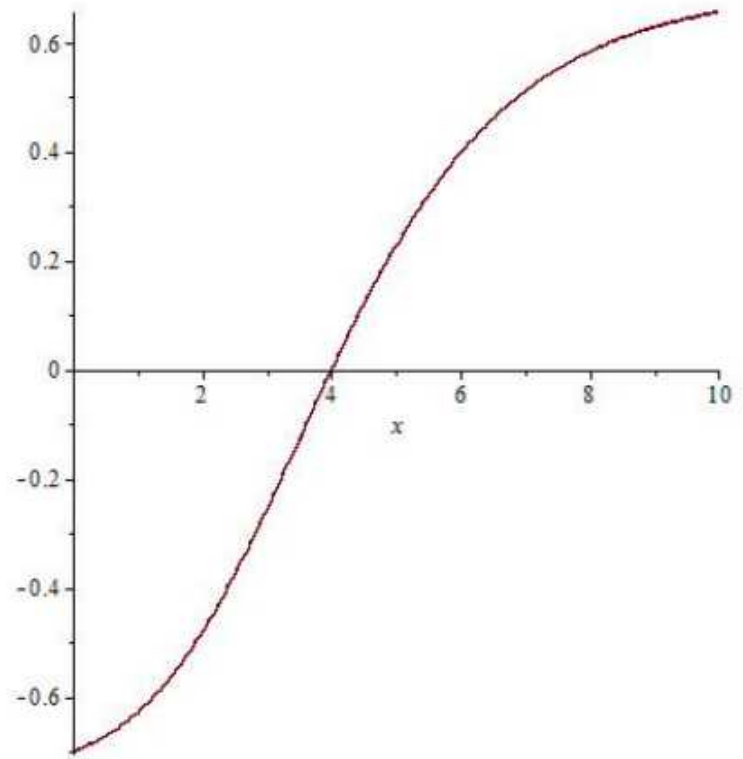

(b) Real 2D Shape 
Please see the Manuscript PDF file for the complete figure caption

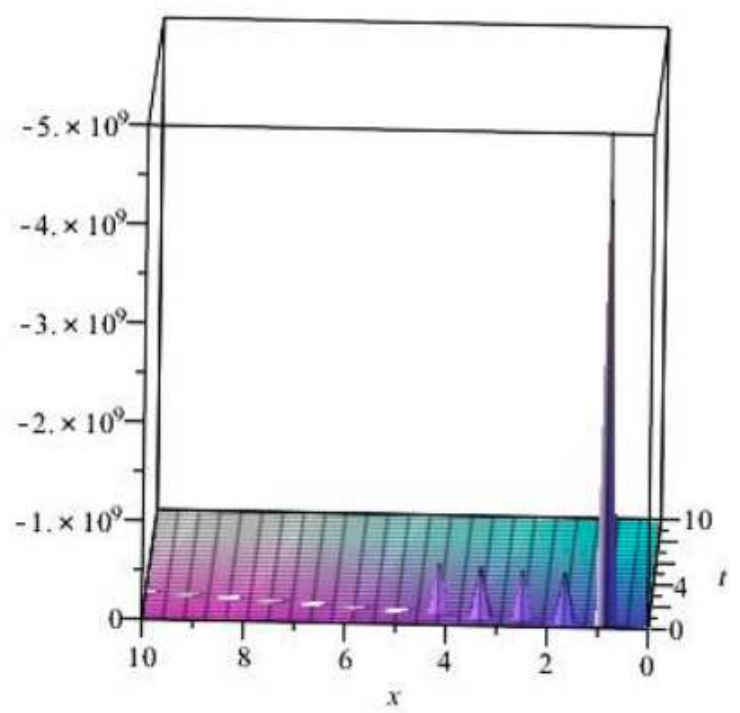

(a) Real 3D surface

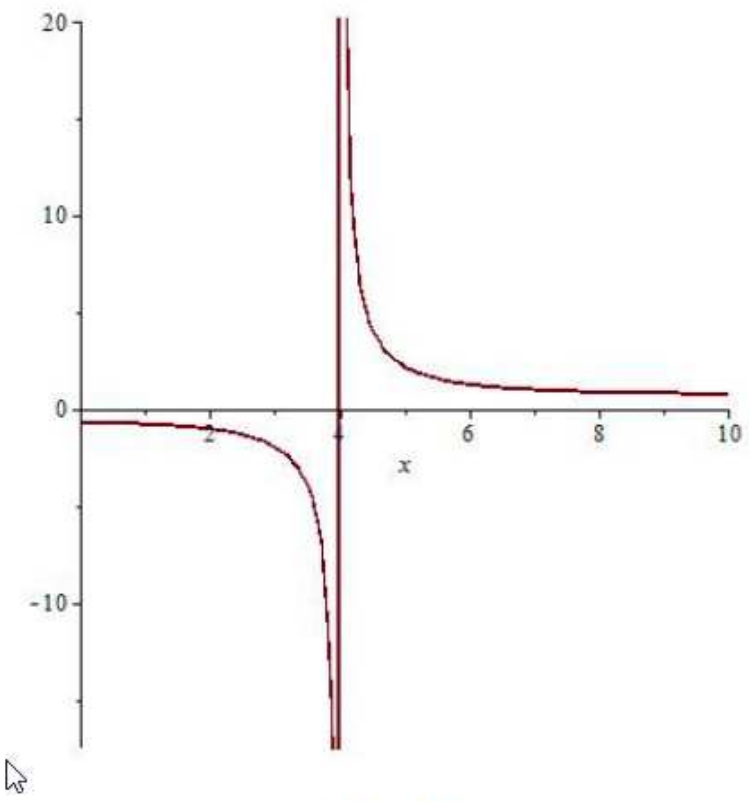

(b) Real 2D Shape

\section{Figure 3}

Please see the Manuscript PDF file for the complete figure caption

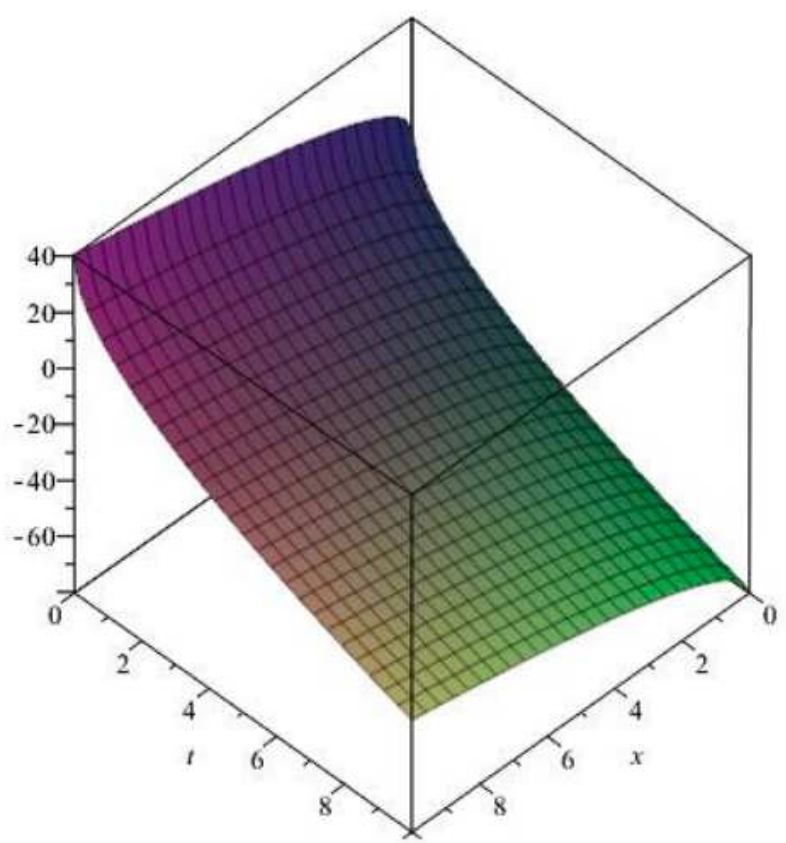

(a) Real 3D surface

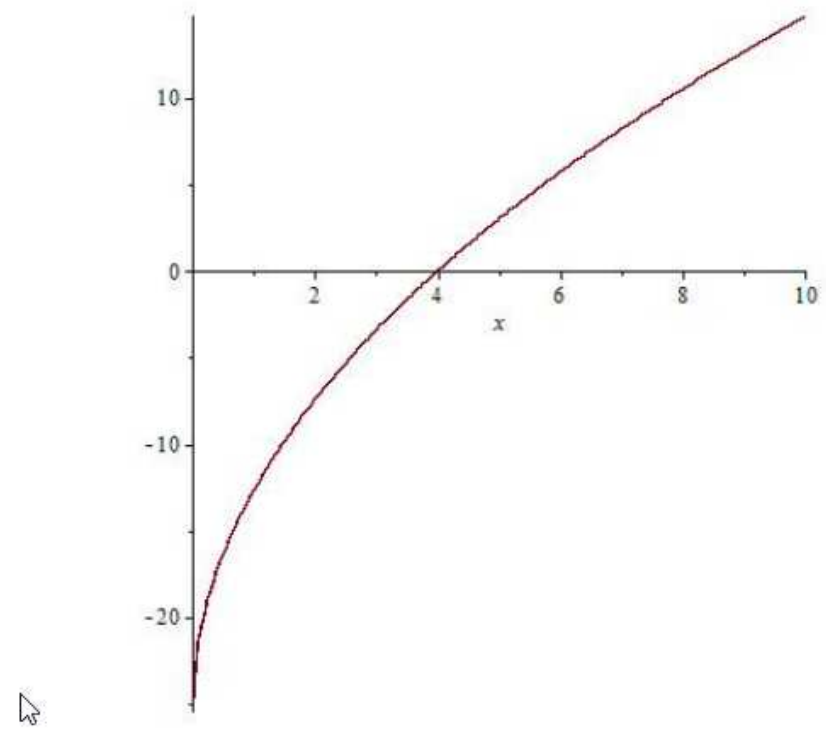

(b) Real 2D Shape

\section{Figure 4}

